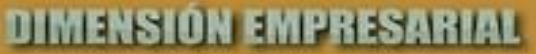

FreE ASSAY

\section{COOPETENCIA, INTERACTION OF TWO ANTAGONIST RELATIONS ${ }^{i}$}

LA COOPETENCIA, INTERACCIÓN DE DOS RELACIONES ANTAGÓNICAS

COOPETENCIA, INTERACÇÃO DE DUAS RELAÇÕES ANTAGONAS

\section{CITATION}

Javier Gómez Diazii

Alejandro García Garnica iii

Gustavo Curiel Avilésiv

Gómez-Diaz; Javier; García-Garnica, Alejandro \& Curiel-Avilés, Gustavo (2019). Coopetencia, interaction of two antagonist relations. Dimensión Empresarial, 17(1), 130-137. DOI: http://dx.doi.org/10.15665/dem.v17i1.1506. ID: http://ojs.uac.edu.co/index.php/dimension-empresarial/issue/view/99

\begin{abstract}
Businesses are developed in highly dynamic environments, so companies seek access to resources that allow them to cope with variations. One of these strategies is cooperation in which companies cooperate and compete towards a common goal. In the cooperative, companies that compete and cooperate looking for a common goal. Through sharing (cooperation) the resources create value, the appropriation of this value is derived from the various interests (competition) of the companies, catches the value created for their purposes.
\end{abstract}

Key words: Coopetencia, competitive advantages

\section{RESUMEN}

Las empresas se desarrollan en entornos altamente dinámicos, por lo cual ellas buscan acceder a recursos que les permitan hacer frente a las variaciones. Una de estas estrategias es la coopetencia en la cual las empresas cooperan y compiten hacia un objetivo en común. En la coopetencia, las empresas compiten y cooperan buscando un objetivo en común. A través de compartir (cooperación) recursos crean valor, la apropiación de este valor se deriva de los diversos intereses (competencia) de las empresas, las cuales captan el valor creado para sus propósitos.

Palabras clave: cooperación, ventaja competitiva

\section{RESUMO}

As empresas são desenvolvidas em ambientes altamente dinâmicos, pelo que as empresas buscam o acesso a recursos que lhes permitem lidar com as variações. Uma dessas estratégias é a co-opção em que as empresas cooperam e competem em direção a um objetivo comum. Na coopetencia, as empresas competem e cooperam procurando um objetivo comum. Através dos recursos de partilha (cooperação) criam valor, a apropriação deste valor deriva dos vários interesses (concorrência) das empresas, que capturam o valor criado para os seus fins.

Palavras-chave: coopetencia, vantagem competitiva 


\section{INTRODUCCIÓN}

El entorno económico dinámico en el que están inmersas las empresas requiere formas alternas de realizar negocios con el fin de que las organizaciones logren sus objetivos. Las firmas, examinan estrategias para mejorar sus servicios y/o productos, disminuir costos, participar en la gestión de conocimientos, etc. con lo cual buscan generar ventajas competitivas. Sin embargo, en muchas ocasiones las empresas no pueden por si solas, promover procesos que les permitan competir en los mercados que participan. Por lo anterior, las firmas establecen relaciones de cooperación con instituciones educativas, gobierno, proveedores $y$ empresas (con las que rivalizan en el mercado). Esta última relación es la que llama el interés de los investigadores.

La estrategia de cooperación-competencia constituye una táctica alternativa para las empresas al formar alianzas con diversos competidores y buscar recursos complementarios para aumentar las capacidades en la búsqueda de oportunidades de negocio. Esta relación de cooperacióncompetencia, son denominadas, como coopetencia. En esta las compañías entran a una nueva dinámica en lo que respecta a las relaciones empresariales, ya que en la coopetencia se unen dos relaciones empresariales que son antagónicas. Sin embargo, estos vínculos coexisten al momento de buscar un beneficio en común. A través de la coopetencia las empresas están llamadas a iniciar acciones colectivas con sus competidores para crear valor en el mercado y, en el mismo tiempo compiten para captar el valor creado individualmente. Por lo anterior, el objetivo del presente trabajo es analizar los conceptos básicos para entender la coopetencia interempresarial y su dinámica para crear y apropiar valor.

\section{LA COOPETENCIA INTEREMPRESARIAL}

Para entender el tema es necesario considerar lo que mencionan Brandenburger \& Nalebuff (2005). estos autores explican que es común que la visión de la empresa respecto a los negocios sea en un sentido de guerra, considerar a todos las demás empresas como competencia y no como complemento. Sin embargo, es de notar que

hay cooperación cuando los proveedores, las compañias y los clientes se juntan para crear valor; pero cuando se trata de repartir la torta, los clientes presionan para obtener precios más bajos y los proveedores también quieren su tajada. De modo que a la hora de dividir la torta hay competencia (Brandenburger y Nalebuff, 2005, p. 53).
Esto quiere decir que las empresas son complementadoras para crear mercados y competidoras al dividirlos.

Brandenburger \& Nalebuff (2005, p. 24) desarrollaron un modelo que se denomina Neto para representar las relaciones e interdependencias entre los diferentes jugadores de un juego de negocios (figura 1). El valor neto constituye una red de relaciones coopetitivas en que las organizaciones juegan múltiples funciones y buscan socios complementarios para crear valor. En el centro de la red de valores se coloca a la empresa en cuestión. En el eje vertical se encuentran los proveedores y los clientes que contribuyen igualmente al proceso de creación de valor. En el eje horizontal están los competidores y las empresas complementarias. Los competidores son empresas que pueden sustituir a la empresa central, ya sea mediante la venta a sus clientes, o mediante la compra a sus proveedores. Mientras que las empresas complementarias se definen como jugadores cuyos productos se aprecian más cuando se combinan con los productos de la empresa central.

La descripción de la competencia y la cooperación ayuda entender que existen diferentes estrategias que las compañías pueden emprender para aumentar sus niveles de competitividad. Por lo cual, cada empresario elige la opción que considera más adecuada para sus fines (Ojeda, 2010). Cuando los empresarios consideran que tienen similitudes con las empresas que compiten, en lo que se refiere a los desafíos y amenazas del entorno, les permite establecer lazos de cooperación con las empresas y de estas formas pueden competir con más éxito (Tomaszewski, 2013).

De acuerdo con Kossyva, Sarri, Georgopoulus (2014, p. 94), las relaciones de coopeticion consisten en dos dimensiones, la creación de valor y la apropiación de valor. La primera dimensión se debe a las actividades de cooperación, mientras que la segunda se deriva de las actividades competitivas. La creación de valor se deriva de esfuerzos conjuntos con los competidores directos que tienen los recursos y capacidades complementarias y relevantes. La apropiación de valor se deriva de diversos intereses en que las empresas compiten en captar el valor creado para los propósitos individuales. Al mismo tiempo, compitiendo las empresas tratan de explotar los recursos adquiridos a través de la cooperación en función de sus conocimientos y su capacidad de absorción para utilizarlos a futuro como ventaja competitiva. Por lo anterior, la cooperación y la competencia son importantes para el buen funcionamiento de muchos sistemas económicos. (Cason y Gangadharan, 2013). 
Figura 1. Red de valores

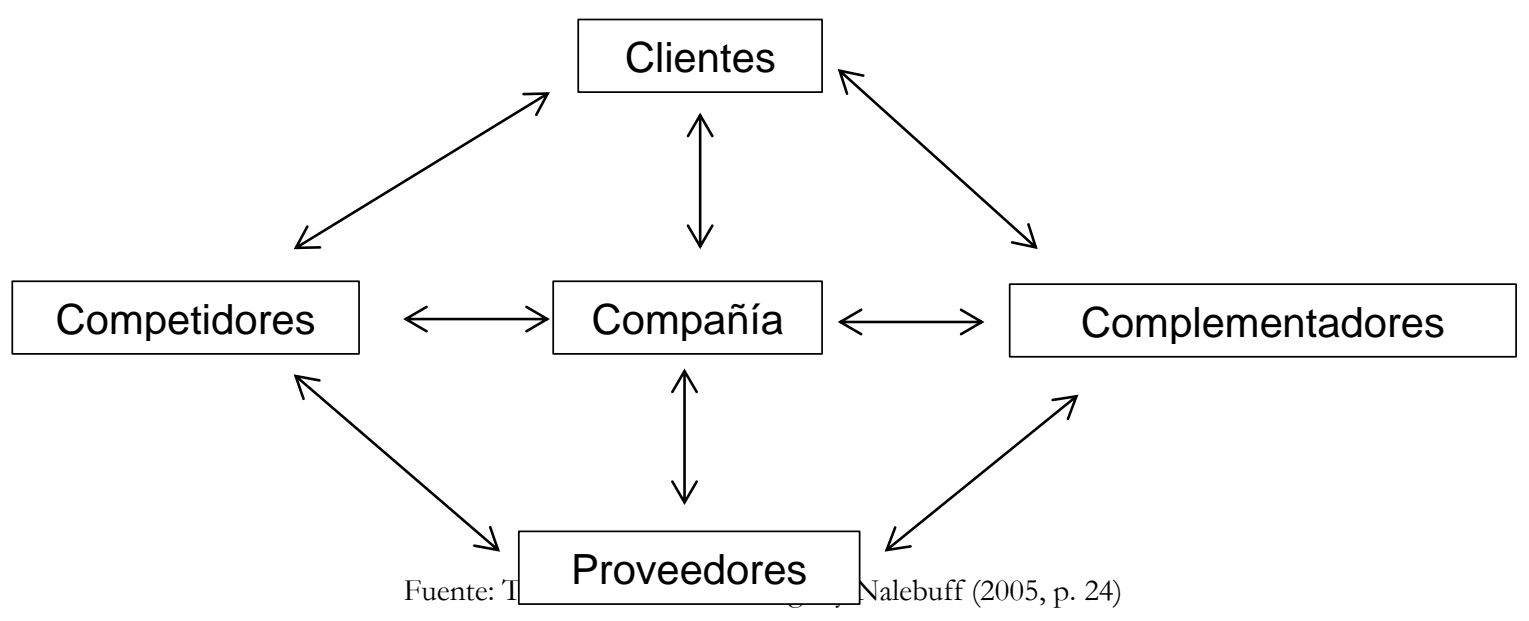

Por otra parte, en lo que se refiere a la definición de la coopeticion, para Crisan (2013, p. 82)

la coopeticion se define como el comportamiento hibrido que comprende la cooperación-competencia. Dependiendo del nivel de análisis, las partes cooperativas y competitivas son parte de un continuo (individual, equipo y organizacional) o están separados por actividad $y / 0$ limites espaciales (interorganizacionales y de red).

Otra definición de este término es el propuesto por Chin, Chan y Lam (2008, p. 438)

la coopetencia es una combinación de la cooperación y competencia. La coopeticion crea valor a través de la cooperación de empresas que compiten, alineando intereses bacia un objetivo comin y ayuda a crear oportunidades para generar ventajas competitivas mediante la eliminación de barreras externas y neutralizar las amenazas. El objetivo central y primordial es crear intercambios para crear valores.

Por su parte, Luo, Slotegraaf y Pan, (2006, p. 68) proponen que la coopetencia

es la ocurrencia conjunta de la cooperación y competencia. Al considerar ambos tipos de relaciones, la investigación muestra que tienen mayor valor cuando existe una combinación de ambas formas. Por lo tanto, se espera que las empresas mejoren su rendimiento cuando exhiben lazos sociales de cooperación que están anidados en un marco competitivo más amplio.

Branderburger y Nalebuff (2005, p. 54) explica que

la coopetencia se define como la combinación de cooperación y competencia para la creación de valor. La coopetencia se basa en la teoría de juegos, donde el negocio es un juego con múltiples jugadores (empresas) que juegan múltiples funciones y dependen unos de otros.

Un juego de los negocios es diferente de los deportes o guerra donde hay ganadores y perdedores. Participar en un juego de los negocios, las empresas pueden obtener más beneficios al final, como su interacción podría generar la suma positiva en el juego. Esto significa que el éxito de una empresa no significa necesariamente el fracaso de los demás.

Considerando las cuatro definiciones anteriores se puede puntualizar a la coopetencia como la relación entre empresas que cooperan y compiten simultáneamente hacia un objetivo en común. De esta forma, la coopeticion

se produce cuando dos empresas cooperan en algunas actividades de negocio a la vez que compiten entre sí. Por lo tanto, los elementos de cooperación y competencia están presentes. Esto significa, que dos empresas podrian cooperar coordinando sus operaciones de compra y aprovisionamiento de servicios, al mismo tiempo que están compitiendo en las áreas de fabricación y comercialización (Morris, Kocak y Ozer, 2007, p. 36). 


\section{JAvier Gómez Diaz, ALejandro García GARniCA y Gustavo Curiel AviLés}

Cuadro 1. Modelo de los diferentes tipos de coopeticion

\begin{tabular}{|c|c|c|c|}
\hline \multirow{3}{*}{ 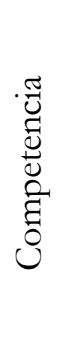 } & \multirow{3}{*}{$\begin{array}{l}\text { Alta } \\
\text { Baja }\end{array}$} & $\begin{array}{l}\text { Contendiente } \quad \text { Tipo } 2 \\
\text { (alta competencia, baja cooperación) }\end{array}$ & $\begin{array}{l}\text { Adaptativo } \quad \text { Tipo } 4 \\
\text { (alta competencia, alta cooperación) }\end{array}$ \\
\hline & & $\begin{array}{l}\text { Monoplayer } \quad \text { Tipo } 1 \\
\text { (baja competencia, baja cooperación) }\end{array}$ & $\begin{array}{l}\text { Compañero } \quad \text { Tipo } 3 \\
\text { (baja competencia, alta cooperación) }\end{array}$ \\
\hline & & Cooperación & Alto \\
\hline
\end{tabular}

Fuente: Chin, Chan y Lam (2008, p. 439)

Por otro lado, Chin, Chan y Lam (2008), considerando que la intensidad con la que colaboran y compiten las empresas no es siempre la misma, y que esta fuerza de relación puede ir de baja a alta intensidad con lo que estos autores establecen cuatro tipos de coopeticion (Cuadro 1).

De esta tipología propuesta en el cuadro 1, los mismos autores explican que en cada tipo de relación resultado de la cooperación competencia, se dan diferentes resultados que se explican a continuación:

$\checkmark$ El tipo monoplayer (bajo nivel de competencia, bajo nivel de cooperación) no interactúa significativamente con los competidores, manteniendo un bajo grado de competencia y bajo grado de cooperación con los competidores.

$\checkmark \quad$ Contendiente (alta competencia, baja cooperación). Es una empresa que vive con competidores para mantener su posición en el mercado. $\checkmark$ Compañero (bajo nivel de competencia, máxima cooperación) mantiene un alto grado de cooperación y bajo grado de competencia, busca sinergias para obtener recursos y capacidades complementarias

$\checkmark$ Adaptativo (alta competencia, alta cooperación) las empresas buscan alcanzar metas conjuntas manteniendo un alto grado de cooperación y competencia. (Chin, Chan y Lam, 2008: 439 y 440).

Así como existen diferentes tipos de coopetición de acuerdo con la intensidad de la relación cooperacióncompetencia, también existen fluctuaciones en el tiempo. Este tema es abordado por Crisan (2013), este autor explica estas relaciones mediante una gráfica (Figura 2), donde se ha representado la relación coopetitiva, considerando la intensidad (eje y) y la duración (eje x) de la competencia/cooperación pueden variar.

Figura 2. Dinamica de la coopeticion en el tiempo

\section{Competencia}

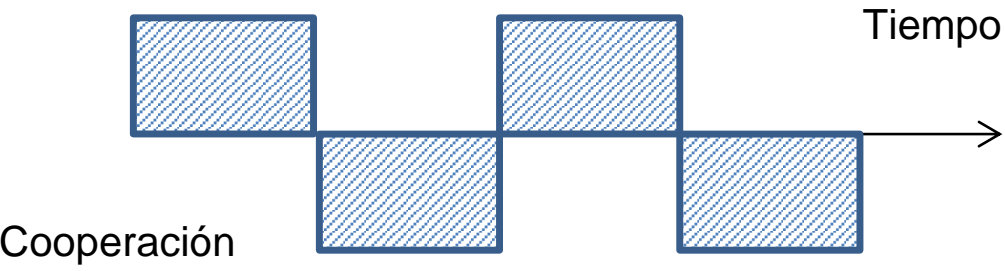

Fuente: Crisan (2008, p. 81)

En lo que se refiere a los factores que promueven la coopetencia, Morris, Kocak y Ozer, (2007, p.37) mencionan que:

cuando las actividades de las empresas se relacionan a una mayor distancia de los compradores, las empresas tienden a cooperar. Cuando las actividades están cerca de los compradores las empresas tienden a competir. Esto también sucede cuando la empresa tiene ciertos recursos en su poder se da la competencia; sin embargo, cuando se decida compartir recursos que no comprometa su posición competitiva la cooperación se hace fuerte.

La coopetencia trae beneficios a las empresas participantes, como menciona Parra, García y Jiménez (2008, p. 88)

en las relaciones coopetitivas las empresas interactúan de acuerdo con dos lógicas diferentes de interacción, la cooperación y la competencia. De este modo, en la parte cooperativa de la relación las empresas ganarán acceso a recursos externos, tales como know-how o fondos 
financieros, mientras que en la parte de competencia las empresas están forzadas a generar una ventaja competitiva relativa sobre los otros actores. Consecuentemente, las relaciones coopetitivas incluirán simultáneamente los beneficios e inconvenientes de la cooperación y la competencia”.

De acuerdo con Morris, Kocak y Ozer (2007, p. 37)

la coopetencia con lleva tanto beneficios como costos. Las empresas participantes. En términos de beneficios, tiene un efecto positivo sobre el rendimiento del negocio, acceder a fuentes externas de conocimiento, aprendizaje organizacional especialmente en lo que se relaciona con las competencias. Dentro de los costos se encuentran costos financieros, pérdida de control de actividades o recursos clave.

\section{ABORDAJES EMPÍRICOS SOBRE LA COOPETENCIA}

Diversos investigadores han abordado el tema de la coopetencia. Sin embargo, no existen muchos trabajos que analicen a nivel inter-empresa. Por lo cual, en este documento se consideran algunos de otras temáticas con la finalidad de conocer la forma operativa del análisis de la coopetencia. Algunos han realizado su análisis a nivel de individuos como es el trabajo de Puurtinen y Mappes (2009), quienes a través de un experimento con grupos de individuos buscaron estudiar el efecto de la competencia entre grupos, la percepción de los miembros del grupo tanto competidores y colaboradores, así como las emociones morales: la ira hacia los miembros cuando donan menos y la culpabilidad cuando se gana más que los otros miembros. Estos autores mencionan que a una mayor competencia entre grupos aumenta la cooperación hacia adentro del grupo (Puurtinen y Mappes, 2009, p. 359).

Otro trabajo que analiza la coopetencia desde una perspectiva individual es el de Cason y Gangadhara (2013), en el cual se examina si los vendedores se coordinan con éxito para financiar un proyecto de investigación conjunto para reducir los costos y como esta colaboración afecta su política de precios. En su trabajo los investigadores explican que los individuos cooperan para financiar un bien público cuando se les da una oportunidad, la cooperación es menos frecuente en entornos donde compiten en un mercado similar. No es significante la conducta de cooperación para reducir la competencia (Cason y Gangadhara, 2013, p. 1728).

Con respecto a los trabajos que se han realizado a nivel intraempresa sobre la coopetencia, se encuentra el trabajo de Luo, Slotegraaf y Pan (2006) quienes analizaron la ocurrencia conjunta de la cooperación y la competencia en todas las áreas funcionales dentro de la empresa. A través de entrevistar informantes clave, concluyeron que la cooperación implica la intensidad y generación del conocimiento y la competencia puede facilitar la transferencia del conocimiento. Ambas pueden crear conocimientos que se pueden traducir en una ventaja competitiva (Luo, Slotegraaf y Pan, 2006, p. 76).

En lo que se refiere al análisis a nivel inter-empresa Ojeda (2010), presentó evidencia sobre la experiencia de veintinueve micros, pequeñas y medianas empresas productoras de bienes o prestadoras de servicios relacionados con las principales áreas ambientales: agua, aire, ruido, suelo, energía y residuos, ante la disyuntiva de competir o cooperar. En su análisis indica que

existe más de una manera para lograr un mismo objetivo. Cada empresario elige la opción que considere más adecuada bajo la consideración de sus propias circunstancias, su disposición o aversión al riesgo, sus objetivos y sus valores. Por tanto, dos empresarios ante una misma situación pueden elegir una alternativa diferente. El reto entonces consiste en saber identificar cuándo es momento de competir o de cooperar (Ojeda, 2010, p. 10).

Otro trabajo que aborda la coopetencia a nivel interempresarial es el realizado por Tena y Comai (2001). Estos autores investigaron los propósitos de la inteligencia en la empresa competidora, cooperativa, neutral e individual. Tena y Comai (2001, p. 7) explicaron que

la inteligencia cooperativa muestra su aplicación en el momento en que uno de los dos competidores normalmente el que tiene la posición dominante, señala su orientación estratégica de cooperar, de manera que el otro pueda optar por poner en práctica esta opción y no se produzca un enfrentamiento directo.

Así mismo, Morris, Kocak y Ozer (2007) realizaron entrevistas a pequeñas empresas, concluyendo en su trabajo de investigación que la coopetencia tiene relación con tres dimensiones beneficio mutuo, confianza y compromiso de la empresa. La coopetencia no es una necesidad, es más bien una estrategia coherente para la mitigación del riesgo y el aprovechamiento de los recursos (Morris, Kocak y Ozer, 2007, p. 48).

Por su parte Crisan (2013) analizó como la competencia y la cooperación coexisten en un espacio temporal. Este autor explica que

los cambios en el balance de la cooperación competencia son atribuidos principalmente al intercambio de conocimientos, esto tiene implicaciones en el lado empresarial para encontrar un equilibrio adecuado en el reparto y protección de la información y la acumulación de la confianza (Crisan, 2013, p. 85).

En cambio, Capellán (2005) determinó los beneficios reales de la cooperación estratégica, tanto inter como intraactores, en contraposición al comportamiento natural de rivalidad y competencia que genera el posicionamiento en el mercado. Este autor explico que

la coopetencia, implica un buen nivel de fluidez. operativa al interior de las redes de valores donde convergen: firmas, clientes, proveedores 


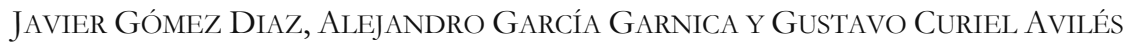

y competidores; así, como la interacción de estos para el diseño de planes estratégicos encaminados, a incrementar los beneficios totales del grupo (Capellán, 2005, p. 288).

En el tema de la aglomeración de empresarial asociado a las relaciones de coopetencia, se encuentra en trabajo de Osarenkhoe (2010). Este autor realizo una investigación que pone de relieve la naturaleza basada en la complementariedad de la estrategia de cooperación competitiva y su impacto en las estrategias colectivas para generar valor entre actores en configuraciones de red. Osarenkhoe (2010, p. 218) determino que

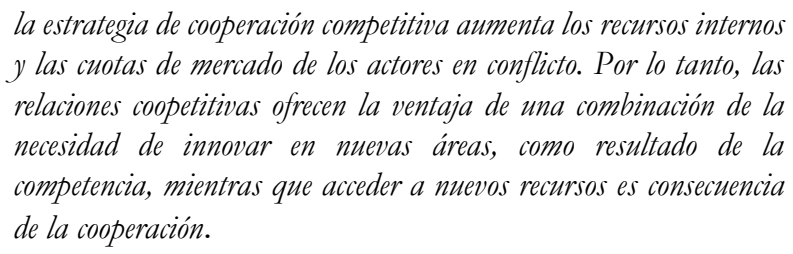
$y$ las cuotas de mercado de los actores en conflicto. Por lo tanto, las relaciones coopetitivas ofrecen la ventaja de una combinación de la necesidad de innovar en nuevas áreas, como resultado de la competencia, mientras que acceder a nuevos recursos es consecuencia de la cooperación.

En un análisis que hacen Parra, García y Jiménez (2008) a nivel de distrito industrial, buscaron desarrollar un modelo explicativo que permita de manera integrada las relaciones de cooperación y competencia que se derivan de la pertenencia a un distrito industrial y su influencia en la generación de ventajas competitivas sostenibles de las empresas del distrito industrial. Estos autores explican en su trabajo que el signo del efecto neto derivado de las relaciones de competencia y de cooperación va a depender de diversas variables contextuales, como el sector de actividad, las características de las empresas pertenecientes y ajenas al distrito (Parra, García y Jiménez, 2008, p. 97).

Por otro lado, Garcia y Lara (2003) plantean en su trabajo que es posible observar y analizar la coexistencia de formas de colaboración y competencia entre distintas empresas tanto a nivel tecnológico y económico, así como al interior de los cluster industriales. Estos autores explican en su trabajo que la cooperación se torna en rivalidad y competencia industrial cuando se juega la propia sobrevivencia de la empresa. Pero la competencia no exime la posibilidad de que empresas rivales lleguen a colaborar de manera conjunta en proyectos (Garcia y Lara, 2003, p. 92).

\section{CONSIDERACIONES FINALES}

Como ha quedado descrito, la coopetencia es una interacción de dos relaciones antagónicas: la cooperación y la competencia. Con la cual, las empresas buscan obtener beneficios que impacten positivamente sobre la competitividad de estas. En la parte cooperativa las empresas ganan accesos a recursos, que por sí solas no podrían conseguir. Y, en la parte de competencia, buscan generar una ventaja competitiva sobre las empresas con las que luchan para satisfacer la necesidad de un mismo cliente.

En la coopetencia, las empresas que compiten cooperan buscando un objetivo en común. A través de compartir recursos para crear valor, que les permita aumentar su rendimiento y generar ventajas competitivas. Cabe destacar que en la coopetencia existen fluctuaciones temporales ya que no en todo momento las empresas buscan coopetir. Si no más bien estos procesos de cooperación-competencia están dados por el entorno económico dinámico que incentivan este proceso.

\section{REFERENCIAS}

Antolín, L. R., Martínez del rio, J. y Céspedes, L. J. (2013). Cooperacion y competencia como antecedentes de la innovación de producto. ¿Aplican las empresas nuevas y establecidas una lógica diferenciada? Investigaciones Europeas de Dirección y Economía de la Empresa, 19, 53-62. DOI: http://dx.doi.org/10.1016/j.iedee.2012.09.001

Beersman, B., Hollenbeck, R. J., Moon, H. y Conlon, D. (2003). Cooperation, competition, and team performance: toward a contingency approac. Academy of Management Journal, 46(5), 572-590.DOI: http:/ /dx.doi.org/10.2307/30040650

Brandenburger, A. M. y Nalebuff, B. J. (2005). Coopetencia. Bogotá, Editorial Norma.

Capellán, M. A. R. (2005). Cooperación y no-cooperación estratégica: efectos sobre la productividad y la competitividad. Ciencia y Sociedad, XXX(2), 275-292.

Cason, N. T. y Gandadhara, L. (2013). Cooperation spillovers and Price competition in experimental markets. Economic Inquiry, 51(3), 1715-1730. DOI: https://doi.org/10.1111/j.1465-7295.2012.00486.x

Crisan, P. (2013). Coopetition structural dynamics. Managerial Chanllenges of the Comtemporary Society, 5, 81-86

Chin, S. K., Chan, L. B., y Lam. K. P. (2008). Identifying and prioritizing critical success factors for coopetition strategy. Industrial Management \& Data, 108(4), 437-454. 
García, G. A. y Lara, R. A. (2003). Cúmulos industriales, competencia y cooperación tecnológica. Administración y Organizaciones, 11, 77-95.

González, A. T. (2009). La PYME mexicana ante la decisión entre cooperar y competir. Asamblea general de ALAFEC. Ecuador. Disponible en: http://www.alafec.unam.mx/memoria_xi.php

Keith, H. L. y Goldman, M. (1961). Competition and non-competition and its relationship to individual and group productivity. Source Sociometry, 24(1), 46-60. DOI: https://doi.org/10.2307/2785928

Kossyva, D., Sarri, K., y Georgopoulos, N. (2014). Co-opetition: a business strategy for SMES in times of economic crisis. South-Eastern Journal of Economics, 1, 89-106.

Lado, A., Boyd, N. y Hanlo, S. (1997). Cooperation, and the search for economic rents: a syncretic model. The Academy of Management Review, 22(1), 110-141

Luo, X., Slotegraaf, J. R. y Pan, X. (2006). Cross functional coopetition: the simultaneous role of cooperation and competition within firms. Journal of Marketing, 70(2), 67-80. doi: https://doi.org/10.1509/jmkg.70.2.67

Morris, H. M., Kocak, A. y Ozer, A. (2007). Coopetition as a small business strategy: implications for performance. Journal of Small Business Strategy, 18(1), 35-55.

Ojeda, J. (2010). La experiencia de la competencia y la cooperación en algunas MIPyME ambientales. Temas de Ciencia y Tecnología, 14(40), 3-12.

Osarenkhoe, A. (2010). A study of inter-firm dynamics between competition and cooperation. A coopetition strategy. Database Marketing \& Customer Strategy Management, 17, 201-221

Parra, R. G., García, V. P. y Jiménez, M. J. (2008). Coopetición y ventaja competitiva en los distritos industriales.

Investigaciones Europeas de Dirección y Economia de la Empresa. 14(1), 85-102. doi: https://doi.org/10.1016/S11352523(12)60013-6

Puurtinen, M. y Mappes, T. (2009). Between group competition and human cooperation. Proceedings. Biological Sciences, 276, 355-360.

Tena, M. J. y Comai, A. (2001). Los propósitos de la inteligencia en la empresa competidora, cooperativa, neutral e individual. Elprofesional de la Información, 10 (5), 4-11.

Tomaszewski, M. (2013). Chosen determinants of coopetition between the industrial companies of the Lubusz region in the 2009-2011 periods. Management, 17(1), 219-232. 


\section{NOTAS}

${ }^{i}$ Ensayo libre desarrollado en el Instituto Tecnológico de Oaxaca, Oaxaca, http://www.oaxaca.tecnm.mx/web/. Fecha de recepción

ii Doctor en Ciencias en Desarrollo Regional y Tecnológico, docente investigador del Instituto Tecnológico de Oaxaca. Correo: gomezdiazjavier@hotmail.com

iii Doctor en Estudios Organizacionales, Máster en Economía y Gestión del Cambio, docente investigador del Instituto Tecnológico de Oaxaca. Correo: agg67@hotmail.com

iv Máster en Desarrollo Regional y Tecnológico, , docente investigador del Instituto Tecnológico de Oaxaca gustavocurielaviles@gmail.com 